\begin{tabular}{|l||}
\hline \multicolumn{1}{|c||}{ AGS Complex Machine Studies } \\
(AGS Studies Report No.372) \\
Studies of Heavy Ion Injection with Linear Coupling (1998) \\
\hline Study Period: April, May 1998 \\
\hline Participants: C. Gardner, L. Ahrens, K. Zeno \\
\hline Reported by: C. Gardner \\
\hline Machine: Tandem, TTB Line, Booster \\
\hline Beam: Au ${ }^{3+}$ \\
\hline Tools: Booster PUE B4, PIP \\
\hline Aim: Studies of Heavy Ion Iniection with Linear Coupling_(1998) \\
\hline
\end{tabular}




\title{
Studies of Heavy Ion Injection with Linear Coupling (1998)
}

\author{
C. Gardner, L. Ahrens, and K. Zeno
}

September 10, 1998

\section{Introduction}

This is the second of two reports on our continuing investigation of the injection of heavy ions into the Booster using linear coupling. In the first report [1] we introduced a new technique for observing the turn-by-turn evolution of the injected beam and we were able to make some qualitative comparisons between the observations and models of the injection process. We continue along the same lines in the present report, making use of newly acquired data and a new version of the PIP program which allows for a quantitative comparision of observations and models. The new data were acquired during the most recent heavy ion run (April 1998) in which ions of Gold were accelerated and delivered to the experimental floor for the HIP program.

\section{Chopping the Beam for Turn-by-Turn Observations}

To observe the turn-by-turn evolution of the beam at injection, it is necessary to chop the beam so that a short pulse corresponding to each turn can be seen on PUE's (PickUp Electrodes) in the Booster ring. The chopper is located upstream of the first 90 degree bend in the TTB line, and consists of two parallel plates with one plate above and the other below the midplane of the beamline. The upper and lower plates are connected respectively to pulsed and DC high-voltage power supplies, and 
the beam is deflected vertically by applying voltages to the plates. In the past, the chopper has been set up so that only a half-turn pulse of beam is transmitted down the TTB line and injected into the Booster; the turn-by-turn evolution of the half-turn can then be observed on the PUE's in the ring. In this mode of operation, beam is transmitted down the line only when the upper plate is pulsed with a voltage which just cancels a DC bias voltage applied to the lower plate. Putting only a half-turn pulse (some $7.5 \mu \mathrm{s}$ ) down the line is, of course, destructive to the Physics programs; they require the full long pulse from the source (typically $500-1000 \mu \mathrm{s}$ ) which corresponds to several tens of turns around the ring. However, the chopper also can be set up so that all of the long pulse is transmitted except for a half-turn portion which may be selected from any part of the long pulse. In this mode, no bias voltage is applied to the lower plate and beam is transmitted down the line except when a deflecting pulse is applied to the upper plate. The deflecting pulse produces a half-turn gap or "hole" in the long pulse that is essentially invisible to the Physics programs and at the same time provides a turn-by-turn signal on the PUE's. We have found that the turn-by-turn evolution of this "hole" is essentially the same as that of a half-turn pulse of beam by itself. This mode of operation allowed us to make several turn-by-turn measurements of the injected beam without interrupting the HIP program.

\section{Linear Coupling Formalism}

Before turning to the PIP program and the turn-by-turn measurements, we review briefly the linear coupling formalism presented in Ref. [2].

\subsection{Evolution of the Beam Ellipsoid at a Given PUE}

We assume that the emittance of the incoming beam at the exit of the C3 inflector is small compared to the acceptance of the Booster. The beam distribution emerging from the inflector travels around the ring to whatever PUE (pickup electrode) we happen to be observing. At this PUE we define the initial beam ellipsoid to be the smallest ellipsoid that contains the initial beam distribution there. Thus, if we let

$$
\xi_{0}=\left(\begin{array}{c}
\mathbf{U}_{0} \\
\mathbf{V}_{0}
\end{array}\right), \quad \mathbf{U}_{0}=\left(\begin{array}{c}
U_{0} \\
U_{0}^{\prime}
\end{array}\right), \quad \mathbf{V}_{0}=\left(\begin{array}{c}
V_{0} \\
V_{0}^{\prime}
\end{array}\right)
$$


and

$$
\mathbf{Z}_{0}=\left(\begin{array}{c}
\mathbf{X}_{0} \\
\mathbf{Y}_{0}
\end{array}\right), \quad \mathbf{X}_{0}=\left(\begin{array}{c}
X_{0} \\
X_{0}^{\prime}
\end{array}\right), \quad \mathbf{Y}_{0}=\left(\begin{array}{c}
Y_{0} \\
Y_{0}^{\prime}
\end{array}\right)
$$

where $U_{0}, U_{0}^{\prime}, V_{0}, V_{0}^{\prime}$ are the initial horizontal and vertical positions and angles (with respect to the closed orbit) of any particle in the beam distribution, and $X_{0}, X_{0}^{\prime}, Y_{0}, Y_{0}^{\prime}$ are the initial positions and angles (also with respect to the closed orbit) of the beam ellipsoid center, then the initial beam ellipsoid at the PUE is defined by

$$
\left(\xi_{0}-\mathbf{Z}_{0}\right)^{\dagger} \mathbf{E}_{0}^{-1}\left(\xi_{0}-\mathbf{Z}_{0}\right) \leq \epsilon_{b}
$$

where $\mathbf{E}_{0}$ is a real, symmetric, positive definite four-by-four matrix, and $\epsilon_{b}$ specifies the emittance. On the nth turn around the Booster, the positions and angles $X, X^{\prime}, Y, Y^{\prime}$ of the center of the beam ellipsoid at the PUE are given by

$$
\mathbf{Z}=\mathbf{T}^{n} \mathbf{Z}_{0}
$$

where

$$
\mathbf{Z}=\left(\begin{array}{c}
\mathbf{X} \\
\mathbf{Y}
\end{array}\right), \quad \mathbf{X}=\left(\begin{array}{c}
X \\
X^{\prime}
\end{array}\right), \quad \mathbf{Y}=\left(\begin{array}{c}
Y \\
Y^{\prime}
\end{array}\right),
$$

and $\mathbf{T}$ is the transfer matrix for one turn around the Booster starting at the PUE. Similarly, the positions and angles of a beam particle at the PUE on the $n$th turn are given by

$$
\xi=\mathbf{T}^{n} \xi_{0}
$$

where

$$
\boldsymbol{\xi}=\left(\begin{array}{c}
\mathbf{U} \\
\mathbf{V}
\end{array}\right), \quad \mathbf{U}=\left(\begin{array}{c}
U \\
U^{\prime}
\end{array}\right), \quad \mathbf{V}=\left(\begin{array}{l}
V \\
V^{\prime}
\end{array}\right) .
$$

The beam ellipsoid at the PUE on the $n$th turn is therefore given by

$$
(\xi-\mathbf{Z})^{\dagger} \mathbf{E}_{n}^{-1}(\xi-\mathbf{Z}) \leq \epsilon_{b},
$$

where

$$
\mathbf{E}_{n}=\mathbf{T}^{n} \mathbf{E}_{0} \mathbf{T}^{\dagger n}
$$

and $\mathbf{Z}$ is given by (4). Equations (4) and (9) give the complete turn-by-turn evolution of the beam ellipsoid at the PUE. 


\subsection{Normal Modes of Oscillation}

Now, as shown in Ref. [2], the components (5) of $\mathbf{Z}=\mathbf{T}^{n} \mathbf{Z}_{0}$ may be written in the form

$$
\mathbf{X}=\mathbf{X}_{1}+\mathbf{X}_{2}, \quad \mathbf{Y}=\mathbf{Y}_{1}+\mathbf{Y}_{2}
$$

where

$$
\begin{gathered}
\mathbf{X}_{1}=d \mathbf{A}^{n} \widehat{\mathbf{X}}_{0}, \quad \mathbf{X}_{2}=\overline{\mathbf{W}} \mathbf{B}^{n} \widehat{\mathbf{Y}}_{0}, \quad \mathbf{Y}_{1}=-\mathbf{W A}^{n} \widehat{\mathbf{X}}_{0}, \quad \mathbf{Y}_{2}=d \mathbf{B}^{n} \widehat{\mathbf{Y}}_{0} \\
\widehat{\mathbf{X}}_{0}=d \mathbf{X}_{0}-\overline{\mathbf{W}} \mathbf{Y}_{0}, \quad \widehat{\mathbf{Y}}_{0}=\mathbf{W} \mathbf{X}_{0}+d \mathbf{Y}_{0}
\end{gathered}
$$

and

$$
\mathbf{X}_{0}=d \widehat{\mathbf{X}}_{0}+\overline{\mathbf{W}} \hat{\mathbf{Y}}_{0}, \quad \mathbf{Y}_{0}=-\mathbf{W} \widehat{\mathbf{X}}_{0}+d \widehat{\mathbf{Y}}_{0}
$$

Here

$$
\begin{aligned}
& \mathbf{A}^{n}=\left(\begin{array}{cc}
\cos n \psi_{1}+\alpha_{1} \sin n \psi_{1} & \beta_{1} \sin n \psi_{1} \\
-\gamma_{1} \sin n \psi_{1} & \cos n \psi_{1}-\alpha_{1} \sin n \psi_{1}
\end{array}\right) \\
& \mathbf{B}^{n}=\left(\begin{array}{cc}
\cos n \psi_{2}+\alpha_{2} \sin n \psi_{2} & \beta_{2} \sin n \psi_{2} \\
-\gamma_{2} \sin n \psi_{2} & \cos n \psi_{2}-\alpha_{2} \sin n \psi_{2}
\end{array}\right)
\end{aligned}
$$

and $\mathbf{T}^{n}$ is given by

$$
\mathbf{T}^{n}=\mathbf{R} \mathbf{U}^{n} \mathbf{R}^{-1}
$$

where

$$
\begin{aligned}
& \mathbf{R}=\left(\begin{array}{cc}
d \mathbf{I} & \overline{\mathbf{W}} \\
-\mathbf{W} & d \mathbf{I}
\end{array}\right), \quad \mathbf{R}^{-1}=\left(\begin{array}{cc}
d \mathbf{I} & -\overline{\mathbf{W}} \\
\mathbf{W} & d \mathbf{I}
\end{array}\right), \quad \mathbf{U}=\left(\begin{array}{cc}
\mathbf{A} & \mathbf{0} \\
\mathbf{0} & \mathbf{B}
\end{array}\right), \\
& \mathbf{W}=\left(\begin{array}{ll}
W_{11} & W_{12} \\
W_{21} & W_{22}
\end{array}\right), \quad \overline{\mathbf{W}}=\left(\begin{array}{cc}
W_{22} & -W_{12} \\
-W_{21} & W_{11}
\end{array}\right), \quad \mathbf{I}=\left(\begin{array}{ll}
1 & 0 \\
0 & 1
\end{array}\right) .
\end{aligned}
$$

These equations show that in each plane, the turn-by-turn motion of the beam ellipsoid center is the superposition of two normal modes of oscillation with tunes, $Q_{1}$ and $Q_{2}$, given by

$$
2 \pi Q_{1}=\psi_{1}, \quad 2 \pi Q_{2}=\psi_{2} .
$$


Furthermore, as shown in Ref. [2], the vectors $X_{1}, X_{2}, Y_{1}, Y_{2}$ are each constrained to lie on an ellipse, so that the motion in each plane is the superposition of motion about two ellipses. The parameters $\alpha_{1}, \beta_{1}, \gamma_{1}, \alpha_{2}$, $\beta_{2}, \gamma_{2}, W_{11}, W_{12}, W_{21}, W_{22}, d, Q_{1}$, and $Q_{2}$ are determined by the one-turn transfer matrix $T$, and can be obtained from the MAD code for the Booster ring.

\subsection{The Beam Envelope}

At any given point in the ring, the maximum extent of the CENTER of . the beam ellipsoid in the horizontal and vertical planes is given by

$$
|X| \leq \sqrt{d^{2} \beta_{1} \epsilon_{1}}+\sqrt{\beta_{x 2} \epsilon_{2}}
$$

and

$$
|Y| \leq \sqrt{\beta_{y 1} \epsilon_{1}}+\sqrt{d^{2} \beta_{2} \epsilon_{2}}
$$

where

$$
\beta_{x 2}=\beta_{2} W_{22}^{2}+2 \alpha_{2} W_{22} W_{12}+\gamma_{2} W_{12}^{2},
$$

and

$$
\beta_{y 1}=\beta_{1} W_{11}^{2}-2 \alpha_{1} W_{11} W_{12}+\gamma_{1} W_{12}^{2} .
$$

These formulae are derived in Ref. [2]. The parameters $\alpha_{1}, \beta_{1}, \gamma_{1}, \alpha_{2}, \beta_{2}$, $\gamma_{2}, W_{11}, W_{12}, W_{21}, W_{22}$, and $d$ are determined by the one-turn transfer matrix at the given point and can be obtained from the MAD code. $\epsilon_{1}$ and $\epsilon_{2}$ are the Courant-Snyder Invariants associated with the two normal-modes of oscillation.

Now consider any particle contained inside the beam ellipsoid at a given point in the ring, and let $\mathbf{E}$ be the four-by-four matrix that defines the ellipsoid at that point. We shall assume that the beam ellipsoid is matched to the lattice, in which case the matrix $\mathbf{E}$ is constant from turn-to-turn at the given point, and the maximum possible horizontal and vertical excursions of a beam particle with respect to the center of the ellipsoid are given by

$$
|U-X| \leq \sqrt{\epsilon_{b} E_{11}}, \quad|V-Y| \leq \sqrt{\epsilon_{b} E_{33}}
$$

where $E_{11}$ and $E_{33}$ are matrix elements of $\mathbf{E}$ and $\epsilon_{b}$. is the beam ellipsoid emittance. 
The beam envelope at a given point in the ring is then defined by the equations

$$
|U| \leq|X|+|U-X| \leq \sqrt{d^{2} \beta_{1} \epsilon_{1}}+\sqrt{\beta_{x 2} \epsilon_{2}}+\sqrt{\epsilon_{b} E_{11}}
$$

and

$$
|V| \leq|Y|+|V-Y| \leq \sqrt{\beta_{y 1} \epsilon_{1}}+\sqrt{d^{2} \beta_{2} \epsilon_{2}}+\sqrt{\epsilon_{b} E_{33}} .
$$

These equations give the maximum possible horizontal and vertical extent of the beam at a given point in the ring.

\section{The New PIP Program}

PIP is the name given to the computer program that collects and analyzes oscilliscope traces of the turn-by-turn signals obtained from a PUE in the Booster ring. The code has been modified so that it can properly analyze the turn-by-turn data for the case in which there is coupling between the horizontal and vertical planes. After acquiring oscilliscope traces of Sum and Difference signals from a given PUE, the program first obtains the position of the injected beam versus turn. A Discrete Fourier Transform of the position-versus-turn data then gives the normal-mode tunes and initial phases associated with the coupled betatron oscillations. Using these as starting values, a function of several parameters is then fitted to the position-versus-turn data.

Using the formulae of the previous section, one can show [3] that the horizontal and vertical positions (with respect to the closed orbit) of the center of the beam ellipsoid at a given PUE on the $n$th turn around the ring are given by the expressions

$$
X_{n}=A_{1} \cos \left(n \psi_{1}+\phi_{1}\right)+A_{2} \cos \left(n \psi_{2}+\phi_{2}\right)
$$

and

$$
Y_{n}=B_{1} \cos \left(n \psi_{1}+\eta_{1}\right)+B_{2} \cos \left(n \psi_{2}+\eta_{2}\right)
$$

where $\psi_{1}=2 \pi Q_{1}$ and $\psi_{2}=2 \pi Q_{2}$. The motion in each plane is the superposition of two modes of oscillation, and the tunes $\left(Q_{1}, Q_{2}\right)$, amplitudes $\left(A_{1}, A_{2}, B_{1}, B_{2}\right)$ and phases $\left(\phi_{1}, \phi_{2}, \eta_{1}, \eta_{2}\right)$ are the 10 parameters that characterize the oscillations. These parameters are not all independent; the amplitudes and phases are each functions of only 4 
independent parameters, the initial positions and angles $X_{0}, X_{0}^{\prime}, Y_{0}, Y_{0}^{\prime}$ of the beam ellipsoid center at the PUE. Thus, there are only 6 independent parameters $\left(Q_{1}, Q_{2}, X_{0}, X_{0}^{\prime}, Y_{0}, Y_{0}^{\prime}\right)$ and these ultimately are the parameters one wants to obtain from an analysis of the position-versus-turn data. For data obtained from a horizontal PUE, the PIP program fits equation (27) to the position-versus-turn data to obtain the parameters $A_{1}, A_{2}, Q_{1}, Q_{2}, \phi_{1}$, and $\phi_{2}$. Similarly, for data obtained from a vertical PUE, equation (28) is fitted to obtain $B_{1}, B_{2}, Q_{1}, Q_{2}, \eta_{1}$, and $\eta_{2}$. From the fitted parameters and the lattice parameters at the PUE, the PIP code then calculates the initial positions and angles at the PUE according to the formulae given in Ref. [3]. The initial positions and angles at the point of injection (i.e. at the exit of the C3 inflector) are then obtained by extrapolating the postions and angles at the PUE back to the point of injection. This requires the transfer matrix between the PUE and the point of injection-the specific formulae are given in Ref. [3].

\section{Turn-by-Turn Measurements of Injected Beam}

We now turn to the measurements made with PIP during the April 1998 HIP run. The data were acquired from horizontal PUE B4 in the Booster.

\subsection{Data Set A}

Careful optimization of injection parameters by $\mathrm{K}$. Zeno during the early part of the HIP run led to settings of the unperturbed (i.e. uncoupled) horizontal and vertical tunes, $Q_{x}$ and $Q_{y}$, and skew quadrupole current, $I$, that were close to those required to produce normal-mode tunes $Q_{1}=4.7$ and $Q_{2}=4.8$. With these normal-mode tunes, the injected beam should return to its initial position every ten turns. To test this, we adjusted $Q_{x}$, $Q_{y}$, and $I$ until the PIP analysis of the Sum and Difference signals from PUE B4 yielded $Q_{1}=4.7$ and $Q_{2}=4.8$. The settings of $Q_{x}, Q_{y}$, and $I$ were respectively $4.73,4.79$, and 6.1 Amps. Figure (1) shows the scope traces of the Sum and Difference signals. Here the oscilliscope was set up to average over several tens of shots (to eliminate noise) and the signals were inverted to make them resemble what one sees for an actual pulse of beam rather than for a "hole" in the beam. The Position-versus-Turn 
data, fitted curve, and fitted parameters obtained by the PIP code are shown in Figures (2) and (3). Here we see that $Q_{1}=4.704, Q_{2}=4.800$, and the beam does indeed return to its initial position every ten turns. The Discrete Fourier Transform of the Postion-versus-Turn data is shown in Figure (4).

\subsection{Data Set AA}

Here we again adjusted $Q_{x}, Q_{y}$, and $I$ until the PIP analysis of the Sum and Difference signals from PUE B4 yielded normal-mode tunes $Q_{1}=4.7$ and $Q_{2}=4.8$. We found that settings of $Q_{x}=4.72, Q_{y}=4.78$ and $I=6.1$ Amps were required. Figure (5) shows the scope traces of the signals (as captured by the PIP Program). The Position-versus-Turn data, fitted curve, and fitted parameters obtained by the PIP code are shown in Figures (6) and (7). Here, again, we see that the beam returns to its initial position every ten turns. The Discrete Fourier Transform of the Postion-versus-Turn data is shown in Figure (8).

\subsection{Data Set B}

Further optimization of injection parameters by K. Zeno produced record intensities of $\mathrm{Au}^{31+}$ beam in the Booster $\left(2.1 \times 10^{9}\right.$ ions accelerated to full energy per Booster cycle with $5.3 \times 10^{9}$ ions available at injection). The settings of $Q_{x}, Q_{y}$, and $I$ in this case were 4.74, 4.77, and 4.6 Amps respectively at the beginning of the beam pulse from Tandem. Zeno found that the efficiency of the injection process was significantly enhanced by ramping the vertical tune to a setting of 4.9 near the end of the beam pulse. Data were taken for this setup with the "hole" chopped in the beam pulse somewhat before the start of the vertical tune ramp. Figure (9) shows the scope traces of the Sum and Difference signals obtained from PUE B4. The Position-versus-Turn data, fitted curve, and fitted parameters obtained by the PIP code are shown in Figures (10) and (11). Here we see that the normal-mode tunes are $Q_{1}=4.728$ and $Q_{2}=4.806$. These are not too far from the values $Q_{1}=4.72=4+18 / 25$ and $Q_{2}=4.80=4+20 / 25$ that will make the injected beam return to its initial position only after every 25 turns. The Discrete Fourier Transform of the Postion-versus-Turn data is shown in Figure (12). 


\section{Coupled Injection Schemes}

To understand why some settings of $Q_{x}, Q_{y}$ and $I$ yielded better injection efficiencies than others during the HIP run, and to find settings that may yield even better efficiencies, we consider several different injection schemes and calculate the number of turns that can be injected in each case.

\subsection{The Basic Injection Process}

The injection process begins at the exit of the C3 Inflector where a local distortion of the closed orbit places the orbit near the inflector septum. If the position of the orbit is held fixed here, then after a certain number of turns have been injected, beam will hit the septum and be lost. To prevent this, the orbit must be moved away by an amount sufficient to keep these turns clear of the septum. With the orbit moved to the new position, a certain number of additional turns may be injected until the orbit must again be moved, still further from the septum. As the orbit is collapsed away from the septum in this way, the incoming beam is injected into a series of phase space layers surrounding the orbit. The process continues until the orbit is so far from the septum that any additional beam injected will be outside the acceptance of the machine.

The total amount of beam that can be injected into the machine will depend on the number of phase space layers and the number of turns that can be injected into each layer. To maximize the number of layers, the incoming beam is positioned as close to the septum as possible (without scraping any beam) and the beam ellipsoid is matched to the machine lattice. The number of turns that can be injected into a given layer will depend on the tunes and the coupling introduced by the skew quadrupoles. By judicious choice of these parameters, and, in particular, by introducing coupling, one can maximize the time that the beam stays clear of the septum in a given layer, thereby maximizing the number of turns that can be injected into the layer. Of course, the introduction of coupling has a price; it increases the size of the vertical envelope of the beam. This means that in dipoles 1,5 , and 7 of each Booster superperiod, where the vertical beta function is large and the vertical aperture is small, one can start to lose beam if too much coupling is introduced. Finding the optimum settings for the tunes and the skew quadrupole current therefore requires keeping track of the vertical beam envelope in the dipoles as well as the 
horizontal beam envelope at the septum.

\subsection{Calculations of Turns Injected for Various Schemes}

We assume that the incoming beam ellipsoid is matched to the coupled machine lattice with $\epsilon_{b}=1.0 \times 10^{-6}$. For the settings of $Q_{x}, Q_{y}$ and $I$ considered below, one then finds that the beam ellipsiod has a horizontal half-width of $\sqrt{\epsilon_{b} E_{11}}=3.3 \mathrm{~mm}$ at the exit of the inflector and a vertical half-width of $\sqrt{\epsilon_{b} E_{33}}=3.6 \mathrm{~mm}$ at the vertical beta maximums in the Booster. The center of the incoming ellipsoid is positioned as close to the outer side of the septum as it can be without scraping the septum, and the closed orbit is initially situated so that the horizontal and vertical positions of the ellipsoid center with respect to the orbit are $10 \mathrm{~mm}$ and zero respectively. The initial horizontal and vertical angles of the ellipsoid center (with respect to the orbit) are then given by equation (152) of Ref.[2]. The septum is assumed to be $1 \mathrm{~mm}$ thick and its inside surface is $47.5 \mathrm{~mm}$ from the center of the beam pipe at the exit of the inflector. The vertical half-aperture in the Booster dipoles is $33 \mathrm{~mm}$.

Using equation (4) we calculate the evolution of the beam ellipsoid center as it travels around the ring, and using equations (25-26) we find the maximum horizontal and vertical extent of the beam at the septum and at the vertical beta maximums in the Booster. This allows us to determine the number of turns that can be injected into a layer and how far the orbit has to be moved to keep the beam from hitting the septum. It also tells us when the vertical envelope becomes too large in the Booster dipoles. We define $X_{\mathbf{C}}$ to be the maximum horizontal excursion (with respect to the closed orbit) of the beam ellipsoid Center on its passes by the septum; $X_{\mathrm{C}}+\sqrt{\epsilon_{b} E_{11}}$ must not exceed $45 \mathrm{~mm}$ in order for the injected beam to be inside the machine acceptance once the closed orbit has collapsed completely at the inflector exit. Similarly, we define $Y_{C}$ to be the maximum vertical excursion of the beam ellipsoid Center at the vertical beta maximums in the Booster; $Y_{\mathrm{C}}+\sqrt{\epsilon_{b} E_{33}}$ must not exceed $33 \mathrm{~mm}$. (We assume that the closed orbit is centered vertically in the dipoles.)

\subsubsection{Scheme A}

We consider first the case in which the normal-mode tunes are the same as those for Data Sets A and AA. For the first 4 layers we take $Q_{x}=4.72$, 
$Q_{y}=4.78, Q_{1}=4.7, Q_{2}=4.8$ and use the MAD code to find the current required in the skew quadrupoles to give the desired normal-mode tunes. A current of 11.4 amps is required for the case of protons with momentum $641.43721 \mathrm{MeV} / \mathrm{c}$; for $\mathrm{Au}^{31+}$ ions the required current is obtained by multiplying by the factor (263.766/641.43721). This gives $I=4.69$

Amps-somewhat smaller than the 6.1 Amps found for the data sets. For the 5th layer we move $Q_{y}$ to 4.90 keeping $Q_{x}$ and $I$ fixed; the MAD code then gives normal-mode tunes $Q_{1}=4.712$, and $Q_{2}=4.908$.

The parameters for each layer are summarized in Table I. Here $X_{\mathrm{CO}}$ and $X_{\mathrm{CO}}^{\prime}$ are the horizontal position and angle of the Closed Orbit at the inflector exit, and $X_{C}$ and $Y_{C}$ are as defined above. The column labeled "Turns" gives the number of turns that can be injected into a given layer before having to move the closed orbit away from the septum.

\begin{tabular}{|c|c|c|c|c|c|}
\hline \multicolumn{6}{|c|}{ Table I: Injection Scheme A } \\
\hline \multicolumn{6}{|c|}{$Q_{x}=4.72, Q_{y}=4.78, I=11.4(4.69)$ Amps } \\
\hline Layer & $X_{\mathrm{CO}}(\mathrm{mm})$ & $X_{\mathrm{CO}}^{\prime}(\mathrm{mrad})$ & Turns & $X_{\mathrm{C}}(\mathrm{mm})$ & $Y_{\mathrm{C}}(\mathrm{mm})$ \\
\hline 1 & 41.82 & 6.52 & 3 & 10.00 & 8.52 \\
2 & 34.18 & 5.33 & 10 & 17.65 & 15.04 \\
3 & 26.53 & 4.14 & 10 & 25.30 & 21.56 \\
4 & 18.88 & 2.94 & 10 & 32.94 & 28.08 \\
\hline \multicolumn{6}{|c|}{ Total Turns Injected $=40$} \\
\hline 5 & $Q_{x}=4.72, Q_{y}=4.90, I=11.4(4.69)$ Amps \\
\hline \multicolumn{7}{|c|}{11.14} & .1 .75 & 7 & 40.71 & 17.12 \\
\hline
\end{tabular}

A total of 33 turns can be injected into layers 1-4. Figure (13) shows the horizontal position of the ellipsoid center in the 4th layer at the exit of the inflector for the first 10 turns around the machine. Here we see that the ellipsoid stays clear of the septum until it returns to its initial position on the 10th turn. Figure (14) shows the horizontal and vertical envelopes of the beam in the 4th layer. Here the upper curves are the horizontal and vertical envelopes for the motion of the ellipsoid center and the lower curves are the horizontal and vertical ellipsoid half-widths defined by equation (24). The plot starts at the exit of the inflector and goes for one turn around the ring (some 202 meters). The total vertical envelope of the beam, defined by equation (26), is the sum of the dashed upper and lower curves. It reaches a maximum of $32 \mathrm{~mm}$ which is almost the full vertical half-aperture $(33 \mathrm{~mm})$ in the Booster dipoles. For the 5th layer, the shift 
in the vertical tune reduces the coupling and keeps the vertical excursions of the ellipsoid center from becoming too large in the Booster dipoles; 7 turns can be injected here. Thus, a total of 40 turns can be injected with this scheme.

\subsubsection{Scheme B}

Here we consider the case in which the uncoupled and normal-mode tunes are $Q_{x}=4.75, Q_{y}=4.77, Q_{1}=4.72=4+18 / 25$, and $Q_{2}=4.80=4+20 / 25$ for the first 3 layers. These settings are close to those of Data Set B for which Zeno was able to obtain record intensities in the Booster. Using the MAD code we find that a skew quadrupole current of 11.06 Amps is required for protons; 4.55 Amps are required for $\mathrm{Au}^{31+}$ ions, which agrees very well with the current required for Data Set B. For the 4 th and 5th layers we move $Q_{y}$ to 4.85 keeping $Q_{x}$ and $I$ fixed; the MAD code then gives normal-mode tunes $Q_{1}=4.737$, and $Q_{2}=4.863$. The parameters for each layer are summarized in Table II.

\begin{tabular}{|c|c|c|c|c|c|}
\hline \multicolumn{6}{|c|}{ Table II: Injection Scheme B } \\
\hline \multicolumn{6}{|c|}{$Q_{x}=4.75, Q_{y}=4.77, I=11.06(4.55) \mathrm{Amps}$} \\
\hline Layer & $X_{\mathrm{CO}}(\mathrm{mm})$ & $X_{\mathrm{CO}}^{\prime}(\mathrm{mrad})$ & Turns & $X_{\mathrm{C}}(\mathrm{mm})$ & $Y_{\mathrm{C}}(\mathrm{mm})$ \\
\hline 1 & 41.82 & 6.55 & 4 & 10.00 & 10.42 \\
2 & 34.18 & 5.36 & 4 & 17.65 & 18.38 \\
3 & 26.54 & 4.16 & 25 & 25.29 & 26.34 \\
\hline \multicolumn{6}{|c|}{ Total Turns Injected =56 } \\
\hline 4 & 18.92 & 2.98 & 8 & 32.88 & 21.21 \\
5 & 11.30 & 1.78 & 15 & 40.49 & 26.12 \\
\hline \multicolumn{6}{|c|}{$Q_{x}=4.75, Q_{y}=4.85, I=11.06(4.55) \mathrm{Amps}$} \\
\hline
\end{tabular}

A total of 33 turns can be injected into layers 1-3. Figure (15) shows the horizontal position of the ellipsoid center in the 3rd layer at the exit of the inflector for the first 25 turns around the machine. Here we see that the ellipsoid stays clear of the septum until it returns to its initial position on the 25th turn. Figure (16) shows the horizontal and vertical envelopes of the beam in the 3rd layer. Here, as before, the upper curves are the horizontal and vertical envelopes for the motion of the ellipsoid center and the lower curves are the horizontal and vertical ellipsoid half-widths. The plot starts at the exit of the inflector and goes for one turn around the 
ring. The total vertical envelope of the beam is the sum of the dashed upper and lower curves; it reaches a maximum of $30 \mathrm{~mm}$ which is most of the vertical half-aperture $(33 \mathrm{~mm})$ in the Booster dipoes.

For the 4th and 5th layers, the shift in the vertical tune reduces the coupling and keeps the vertical excursions of the ellipsoid center from becoming too large in the Booster dipoles; 8 and 15 turns can be injected here. Thus, a total of 56 turns can be injected with this scheme.

\subsubsection{Scheme BB}

Here we consider the case in which the uncoupled and normal-mode tunes are $Q_{x}=4.76, Q_{y}=4.76, Q_{1}=4.72=4+18 / 25$, and

$Q_{2}=4.80=4+20 / 25$ for the first 3 layers; this is the same as Scheme B except that the separation of the unperturbed tunes has been reduced to zero $\left(Q_{x}=Q_{y}=4.76\right)$. Using the MAD code we find that a skew quadrupole current of $11.4 \mathrm{Amps}$ is required for protons; $4.69 \mathrm{Amps}$ are required for $\mathrm{Au}^{31+}$ ions. The tune and skew quadrupole settings for the 4 th and 5 th layers are the same as for Scheme B. The parameters for each layer are summarized in Table III.

\begin{tabular}{|c|c|c|c|c|c|}
\hline \multicolumn{6}{|c|}{ Table III: Injection Scheme BB } \\
\hline \multicolumn{6}{|c|}{$Q_{x}=4.76, Q_{y}=4.76, I=11.4(4.69) \mathrm{Amps}$} \\
\hline Layer & $X_{\mathrm{CO}}(\mathrm{mm})$ & $X_{\mathrm{CO}}^{\prime}(\mathrm{mrad})$ & Turns & $X_{\mathrm{C}}(\mathrm{mm})$ & $Y_{\mathrm{C}}(\mathrm{mm})$ \\
\hline 1 & 41.82 & 6.56 & 4 & 10.00 & 10.80 \\
\hline 2 & 34.18 & 5.37 & 10 & 17.64 & 19.04 \\
\hline 3 & 26.54 & 4.17 & 25 & 25.28 & 27.29 \\
\hline \multicolumn{6}{|c|}{$Q_{x}=4.75, Q_{y}=4.85, I=11.4(4.69)$ Amps } \\
\hline 4 & 18.87 & 2.97 & 8 & 32.96 & 21.65 \\
\hline 5 & 11.22 & 1.76 & 15 & 40.61 & 26.68 \\
\hline \multicolumn{6}{|c|}{ Total Turns Injected $=62$} \\
\hline
\end{tabular}

Comparing these numbers with those of Scheme B we see that by reducing the unperturbed tune separation (and therefore increasing the coupling) we are able to put more turns into layer 2. 


\subsubsection{Scheme C}

This is one of the schemes considered in Ref.[2]. The uncoupled and normal-mode tunes are $Q_{x}=4.823333, Q_{y}=4.843333$, $Q_{1}=4.800=4+12 / 15$, and $Q_{2}=4.866=4+13 / 15$ for the first 2 layers. In this case the beam ellipsoid returns to its initial position every 15 turns. Using the MAD code we find that a skew quadrupole current of $9.2 \mathrm{Amps}$ is required for protons; $3.78 \mathrm{Amps}$ are required for $\mathrm{Au}^{31+}$ ions. For layers 3 and 4 we move $Q_{y}$ to 4.90 keeping $Q_{x}$ and $I$ fixed; the MAD code then gives normal-mode tunes $Q_{1}=4.812$, and $Q_{2}=4.911$. The parameters for each layer are summarized in Table IV. A total of 30 turns can be injected into layers 1 and 2. For the 3rd and 4th layers, the shift in the vertical tune reduces the coupling and keeps the vertical excursions of the ellipsoid center from becoming too large in the Booster dipoles; 11 and 11 turns can be injected here. Thus, a total of 52 turns can be injected with this scheme.

\begin{tabular}{|c|c|c|c|c|c|}
\hline \multicolumn{5}{|c|}{ Table IV: Injection Scheme C } \\
\hline \multicolumn{5}{|c|}{$Q_{x}=4.823333, Q_{y}=4.843333, I=9.2(3.78)$ Amps } \\
\hline Layer & $X_{\mathrm{CO}}(\mathrm{mm})$ & $X_{\mathrm{CO}}^{\prime}(\mathrm{mrad})$ & Turns & $X_{\mathrm{C}}(\mathrm{mm})$ & $Y_{\mathrm{C}}(\mathrm{mm})$ \\
\hline 1 & 36.81 & 5.86 & 15 & 15.00 & 15.41 \\
2 & 29.19 & 4.65 & 15 & 22.62 & 23.24 \\
\hline \multicolumn{5}{|c|}{$Q_{x}=4.823333, Q_{y}=4.90, I=9.2(3.78)$ Amps } \\
\hline 3 & 21.55 & 3.44 & 11 & 30.27 & 20.49 \\
4 & 13.92 & 2.22 & 11 & 37.90 & 25.66 \\
\hline \multicolumn{6}{|c|}{ Total Turns Injected $=52$} \\
\hline
\end{tabular}

\subsubsection{Scheme D}

In this scheme, as in $\mathrm{C}$, the normal-mode tunes are such that the beam ellipsoid returns to its initial position every 15 turns. However, here we take $Q_{x}=4.776666, Q_{y}=4.7566666, Q_{1}=4.800=4+12 / 15$, and $Q_{2}=4.733=4+11 / 15$ for the first 3 layers. Using the MAD code we find that a skew quadrupole current of $9.1 \mathrm{Amps}$ is required for protons; 3.74 Amps are required for $\mathrm{Au}^{31+}$ ions. For layers 4 and 5 we move $Q_{y}$ to 4.70 keeping $Q_{x}$ and $I$ fixed; the MAD code then gives normal-mode tunes $Q_{1}=4.788$, and $Q_{2}=4.688$. The parameters for each layer are summarized in Table V. A total of 34 turns can be injected into layers 1-3. For the 4 th and 5 th layers, the shift in the vertical tune reduces the 
coupling and keeps the vertical excursions of the ellipsoid center from becoming too large in the Booster dipoles; 9 and 19 turns can be injected here. Thus, a total of 62 turns can be injected with this scheme.

\begin{tabular}{|c|c|c|c|c|c|}
\hline \multicolumn{6}{|c|}{ Table V: Injection Scheme D } \\
\hline \multicolumn{5}{|c|}{$Q_{x}=4.776666, Q_{y}=4.756666, I=9.1(3.74)$ Amps } \\
\hline Layer & $X_{\mathrm{CO}}(\mathrm{mm})$ & $X_{\mathrm{CO}}^{\prime}(\mathrm{mrad})$ & Turns & $X_{\mathrm{C}}(\mathrm{mm})$ & $Y_{\mathrm{C}}(\mathrm{mm})$ \\
\hline 1 & 41.81 & 6.58 & 4 & 10.00 & 10.32 \\
2 & 34.19 & 5.38 & 15 & 17.61 & 18.19 \\
3 & 26.58 & 4.18 & 15 & 25.23 & 26.05 \\
\hline \multicolumn{5}{|c|}{$Q_{x}=4.776666, Q_{y}=4.70, I=9.1(3.74)$ Amps } \\
\hline 4 & 18.96 & 2.97 & 9 & 32.85 & 22.89 \\
5 & 11.34 & 1.78 & 19 & 40.47 & 28.20 \\
\hline \multicolumn{6}{|c|}{ Total Turns Injected =62 } \\
\hline
\end{tabular}

\section{Comments and Conclusions}

The results of the PIP analysis of Data Sets A, AA, and B are consistent with the turn-by-turn evolution of the beam ellipsoid calculated for Schemes A and B. The various schemes considered help to explain why some settings of the uncoupled tunes $\left(Q_{x}\right.$ and $\left.Q_{y}\right)$ and skew quadrupole current allow for more injected turns than others. They also confirm $\mathrm{K}$. Zeno's original notion that separating the uncoupled tunes near the end of the injection process keeps the vertical beam envelope from becoming too large in the Booster dipoles.

Many different schemes are possible. A program called "cplinj" was developed to do the calculations for the ones considered here and can readily be used to analyze any others that one might consider. Using the code involves two steps. First the MAD code is run for particular settings of $Q_{x}, Q_{y}$, and $I$; it calculates the parameters for the coupled lattice of the Booster and puts them in a file called "Twiss-couple". The program "cplinj" then reads this file and calculates the layer parameters.

Setting up an injection scheme in practice is not easy. Here are a few suggestions:

1. Set up the Tandem Chopper to chop a half-turn "hole" in the beam pulse. 
2. Using the Booster Orbit Control program, adjust the fast injection 4-bump so that it falls quickly away from the septum after the half-turn "hole" is injected. This will allow the "hole" to survive for many turns (some 40 turns are required for a good PIP analysis).

3. Using the Booster Tune Control and Stopband programs, adjust $Q_{x}$, $Q_{y}$, and $I$ until the PIP analysis of the turn-by-turn signals from a given PUE yields the desired normal-mode tunes.

4. Using the Orbit Control program, adjust the fast injection 4-bump so that the predicted number of turns survive in each layer.

5. Make sure that the beam is centered vertically in the Booster dipoles where the vertical aperture is small; this can be done with the local 3-bumps available in the Orbit Control program.

\section{Acknowledgements}

We would like to thank Rich DiFranco for his patience and perseverance in fixing and maintaining the Booster PUE system. We would also like to thank Jim Niederer for his help and advice in using the MAD code.

\section{References}

1. L.A. Ahrens and C.J. Gardner, "A Study of the Effect of Linear Coupling on the Injection of Iron Ions into the Booster", AGS Studies Report No. 365, November 19, 1997.

2. C.J. Gardner, "Notes on Coupled Motion in a Linear Periodic Lattice and Applications to Booster Injection", AGS/AD/Tech. Note No. 427, February 22, 1996.

3. C.J. Gardner, "Formulae for the PIP Analysis of Linear Coupling", AGS/AD/Tech. Note No. 476, March 30, 1998. 
17:-Apr-98 $\quad$ Figure 1

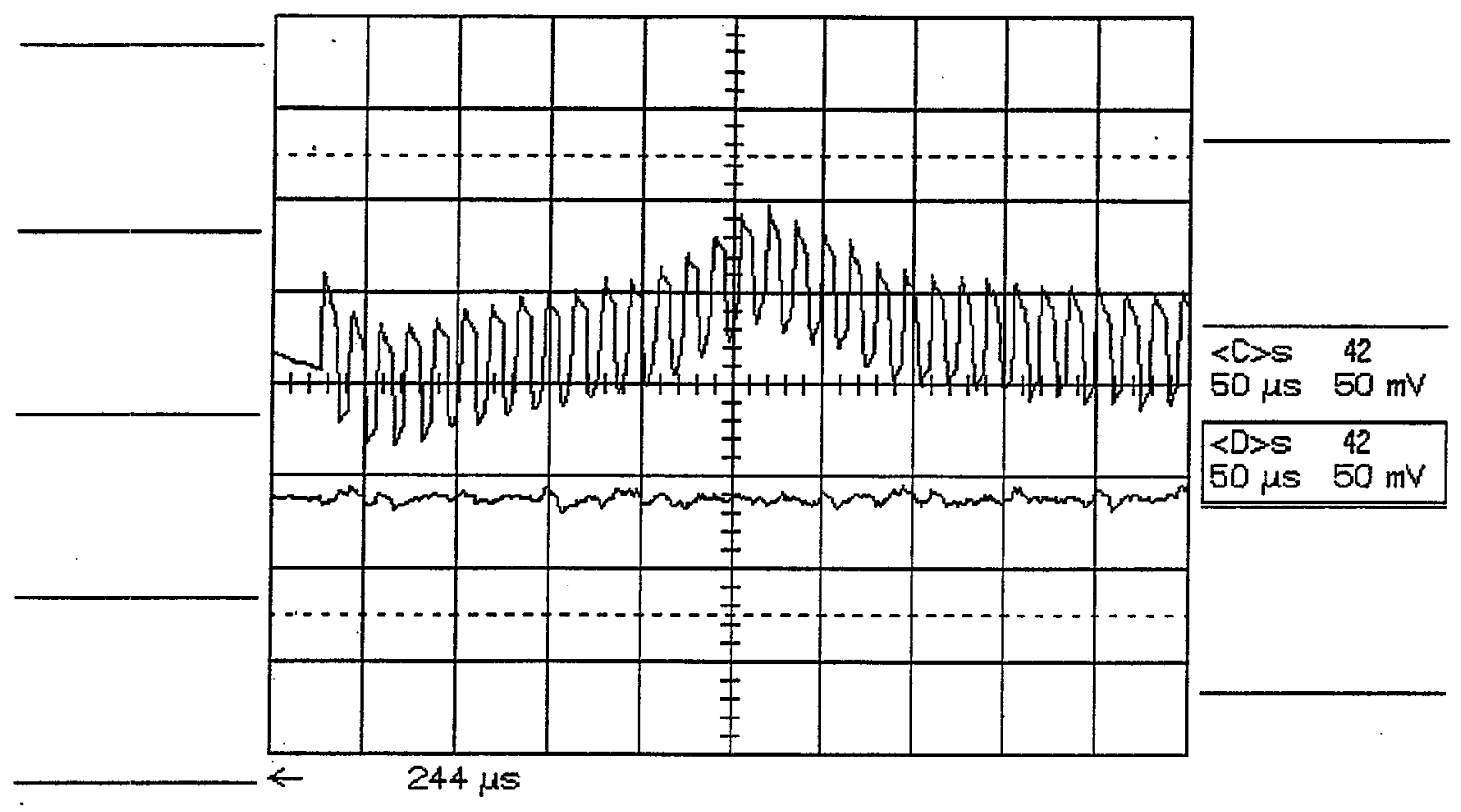

Eigure 2

SelectPue: Booster PUEHB4 - Injection: Booster C3 Inflector $\rightarrow$ |

Thu Apr 9 17:24:04 1998

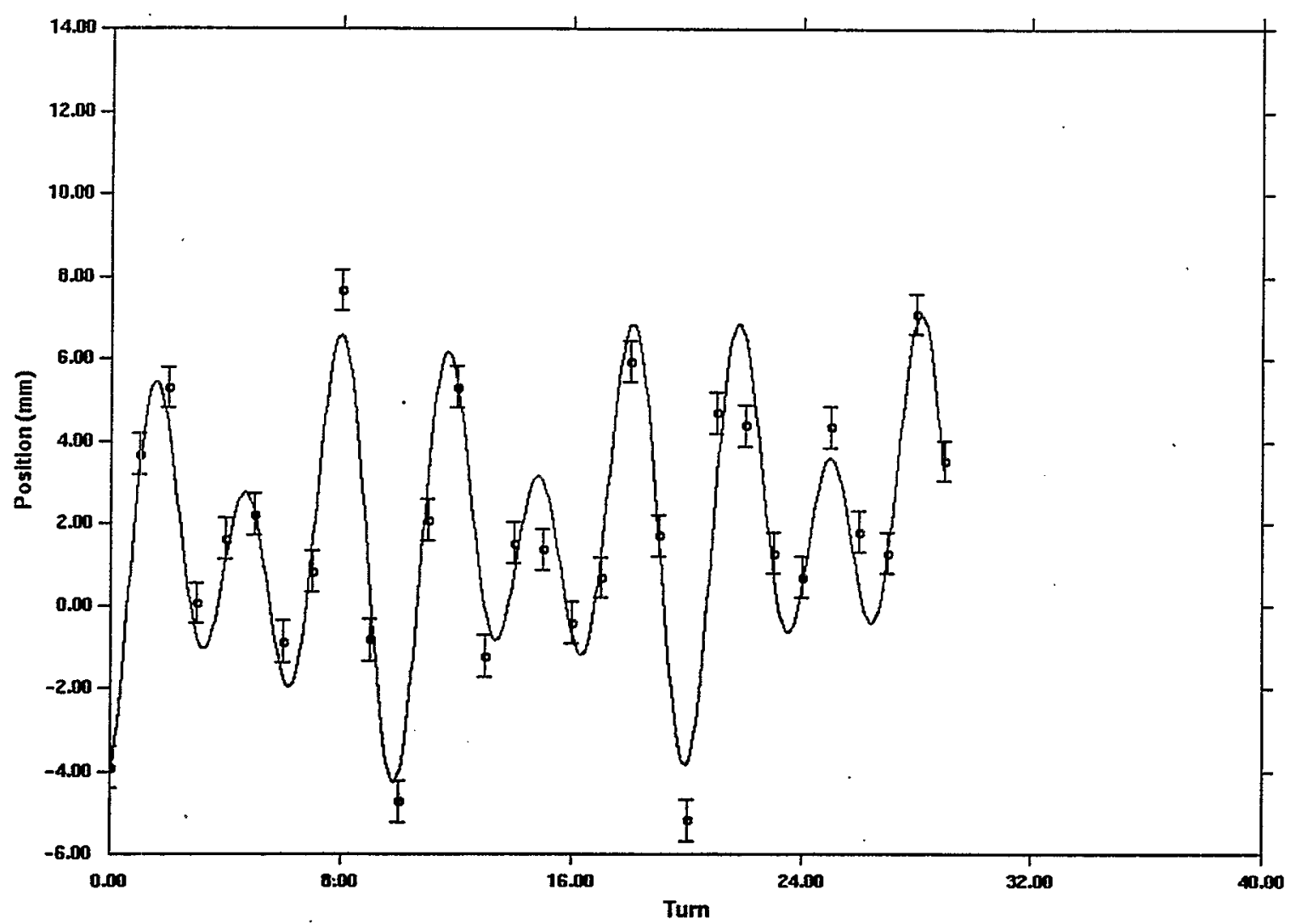




\section{Figure 3}

TRIGGER TIME

: Date $=$ APR 9, 1998, Time $=17: 20: 51.83$

Fitted Parameters: Chisq/dof $=4.717351$, Number of Turns $=30$
$\mathrm{Al}=$
$0.296526+\%-0.000673$
$\mathrm{A} 3=$
$0.595708+/-0.011585$
$\mathrm{A} 2=0.199844+1-0.001117$
$A 4=0.513084+/-0.019264$
$A 6=2.177373+/-0.132524$
$\mathrm{A} 5=3.597105+/-0.131733$

$A 7=1.028306+/-0.180815$

$A 8=0.045885+/-0.010707$

Period $=6.021316$ samples $=1.505329 \mathrm{e}-05$ seconds

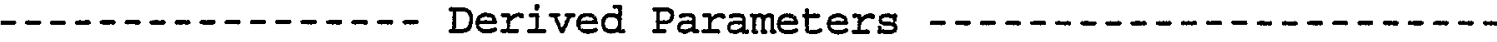

$$
\begin{array}{rlr}
\mathrm{Q} 1 & =4.7035+/-0.0007 \\
\mathrm{Q} 2 & =4.8002+/-0.0011 \\
\mathrm{~A} 1(\mathrm{~mm}) & =3.60+/- & 0.13 \\
\mathrm{~A} 2(\mathrm{~mm}) & =2.18+/- & 0.13
\end{array}
$$

Epsilon 1 (mm mrad) $=1.252676+/-0.118167$

Epsilon $2(\mathrm{~mm}$ mrad) $=1.802917+/-0.272784$

$$
\text { Position of eo at PUE (mm) }=1.03+/-0.18
$$

PUE: BOOSTER PUEHB4 INJ : BOOSTER C3 INFLECTOR

$\mathrm{H}$ Position wrt eo at PUE (mm) $=-5.14+\overline{7}-0.23$

$\mathrm{H}$ Angle wrt eo at PUE (mrad) $=-0.930+/-0.045$

$\mathrm{V}$ Position wrt eo at PUE $(\mathrm{mm})=-1.63+/-0.17$

$\mathrm{V}$ Angle wrt eo at PUE (mrad) $=0.301+/-0.090$

PUE: BOOSTER PUEHB4 INJ: BOOSTER C3 INFLECTOR

$\mathrm{H}$ Position wrt eo at INJ $(\mathrm{mm})=-5.28+7-0.19$

$\mathrm{H}$ Angle wrt eo at INJ (mrad) $=-0.889+/-0.068$

$\mathrm{V}$ Position wrt eo at INJ $(\mathrm{mm})=-1.49+/-0.17$

$\mathrm{V}$ Angle wrt eo at INJ (mrad) $=0.154+/-0.095$

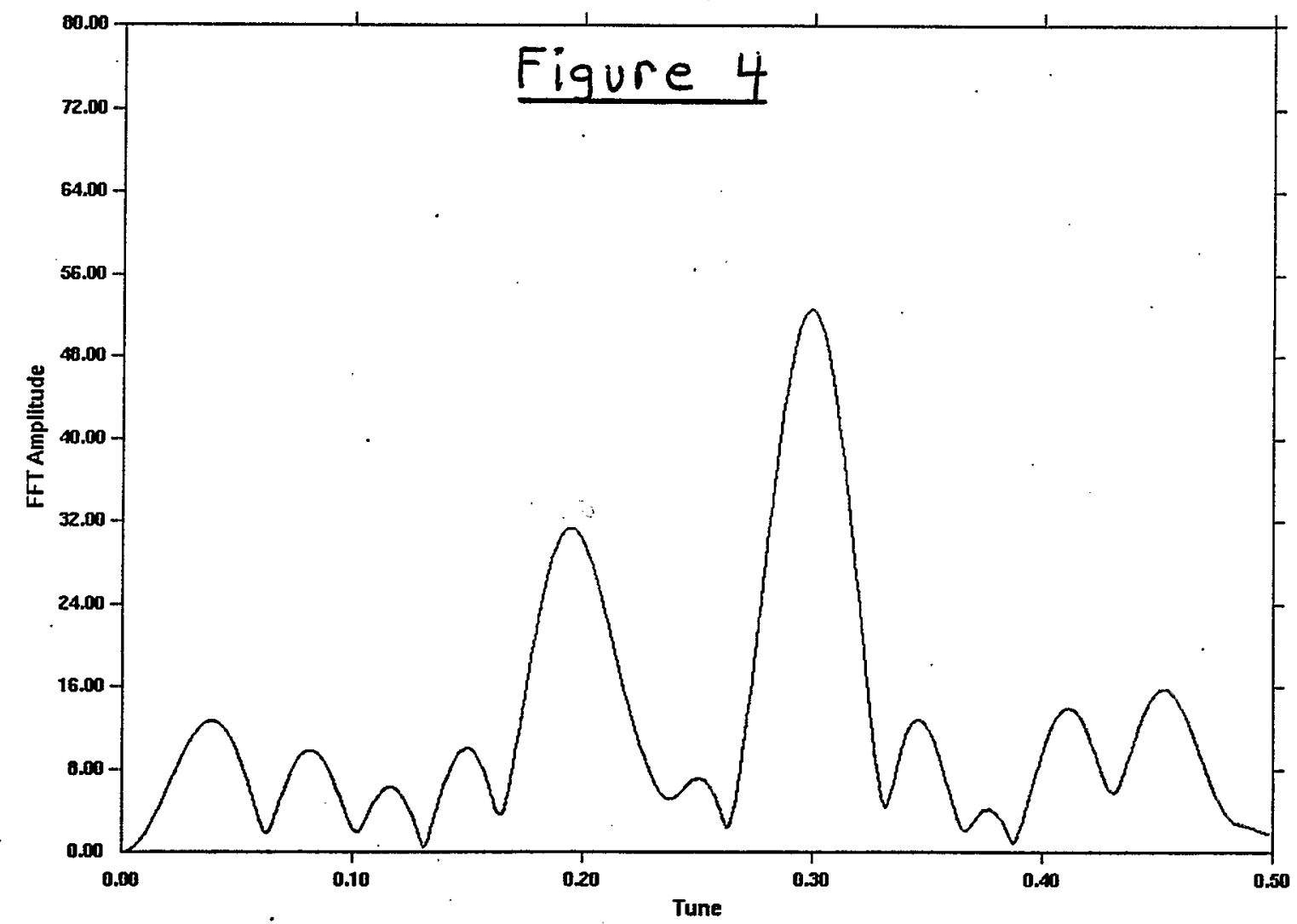


Figure 5
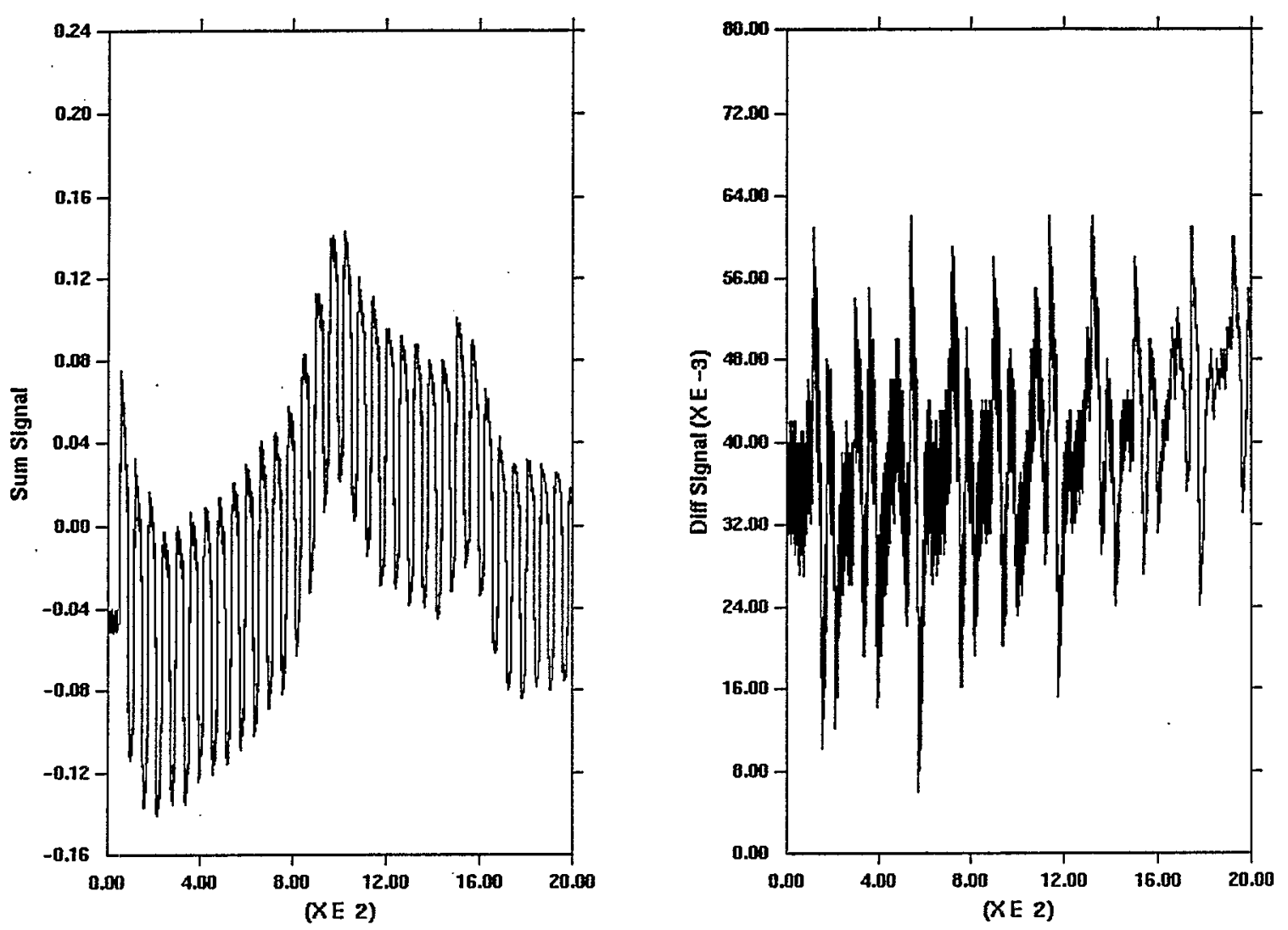

SelectPue: Booster PUEHB4 - Injection: Booster C3 Inflector -1

Sat Apr 25 15:57:40 1998

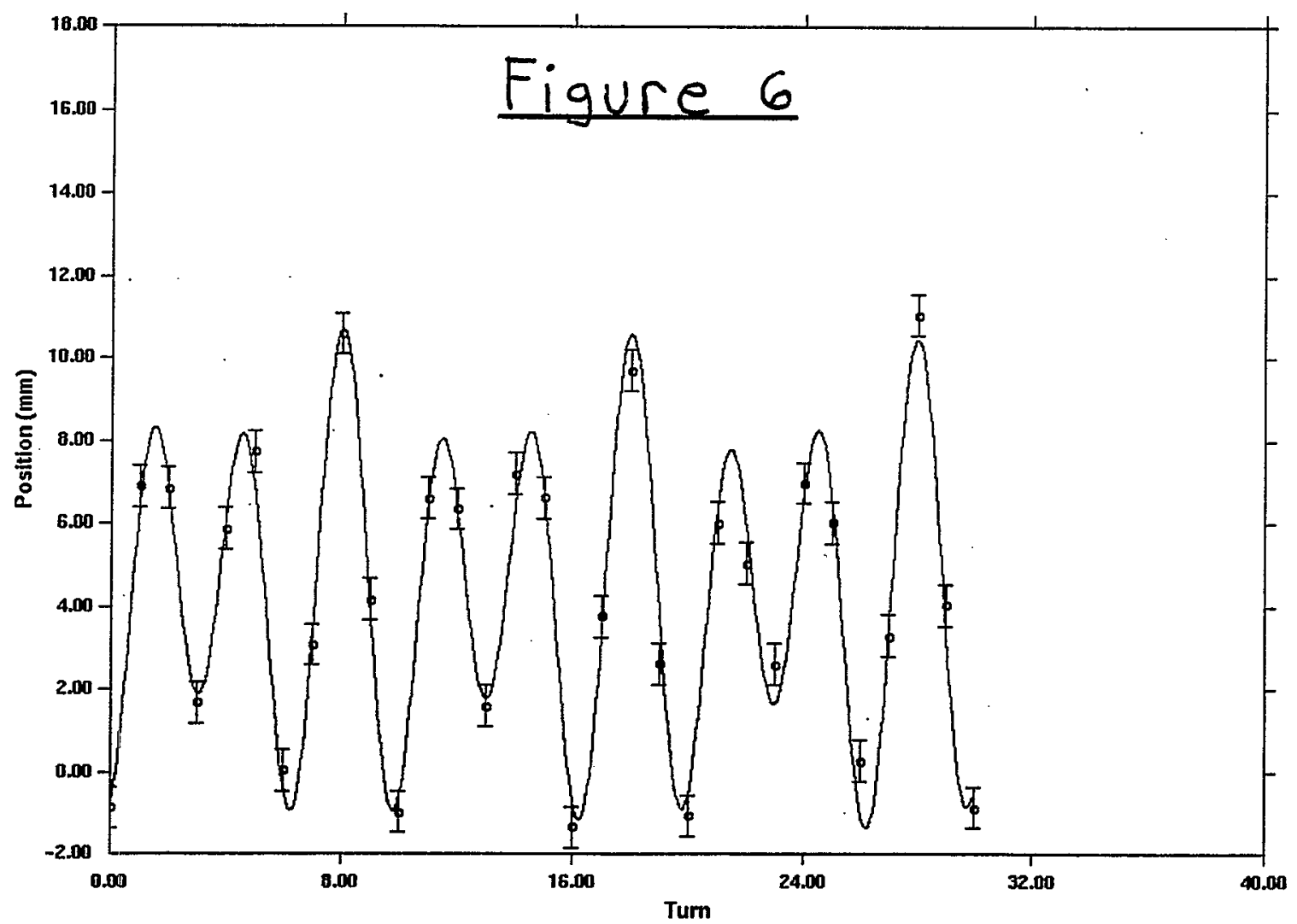




\section{Figure 7}

TRIGGER TIME :Date $=$ APR 25, 1998, Time $=15: 54: 42.83$

Fitted Parameters: Chisq/dof $=1.716329$, Number of Turns $=31$
$\mathrm{A} 1=$
$0.300970+/-0.000527$
$\mathrm{A} 3=$
$0.584275+/-0.009344$
$\mathrm{A} 2=0.199133+/-0.001300$
$A 4=0.400388+/-0.022965$
$A 6=1.788035+/-0.127455$
$A 5=4.436697+/-0.126960$

$A 7=4.561016+/-0.177451$

$A 8=-0.010457+/-0.010202$

Period $=60.235294$ samples $=1.505882 \mathrm{e}-05$ seconds

-.............. Derived Parameters

$Q 1=4.6990+/-0.0005$

$\mathrm{Q} 2=4.8009+/-0.0013$

$$
\begin{aligned}
& A I(\mathrm{~mm})=4.44+/-0.13 \\
& \mathrm{~A} 2(\mathrm{~mm})=1.79+/-0.13
\end{aligned}
$$

Epsilon 1 (mm mrad) $=1.905688+/-0.119578$

Epsilon 2 (mm mrad) $=1.215801+/-0.183142$

$$
\text { Position of eo at PUE }(\mathrm{mm})=4.56+/-0.18
$$

PUE : BOOSTER PUEHB4 INJ: BOOSTER C3_INFLECTOR

$\mathrm{H}$ Position wrt eo at PUE (mm) $=-5.28+\bar{T}-0.25$

$\mathrm{H}$ Angle wrt eo at PUE (mrad) $=-0.873+/-0.043$

$\mathrm{V}$ Position wrt eo at PUE $(\mathrm{mm})=-0.76+/-0.20$

$\mathrm{V}$ Angle wrt eo at PUE (mrad) $=0.540+/-0.065$

PUE: BOOSTER PUEHB4 INJ: BOOSTER C3 INFLECTOR

$\mathrm{H}$ Position wrt eo at INJ $(\mathrm{mm})=-5.48+\bar{T}-0.20$

$H$ Angle wrt eo at INJ (mrad) $=-0.791+/-0.064$

$\mathrm{V}$ Position wrt eo at INJ $(\mathrm{mm})=-0.71+/-0.20$

$V$ Angle wrt eo at INJ(mrad) $=0.352+/-0.072$

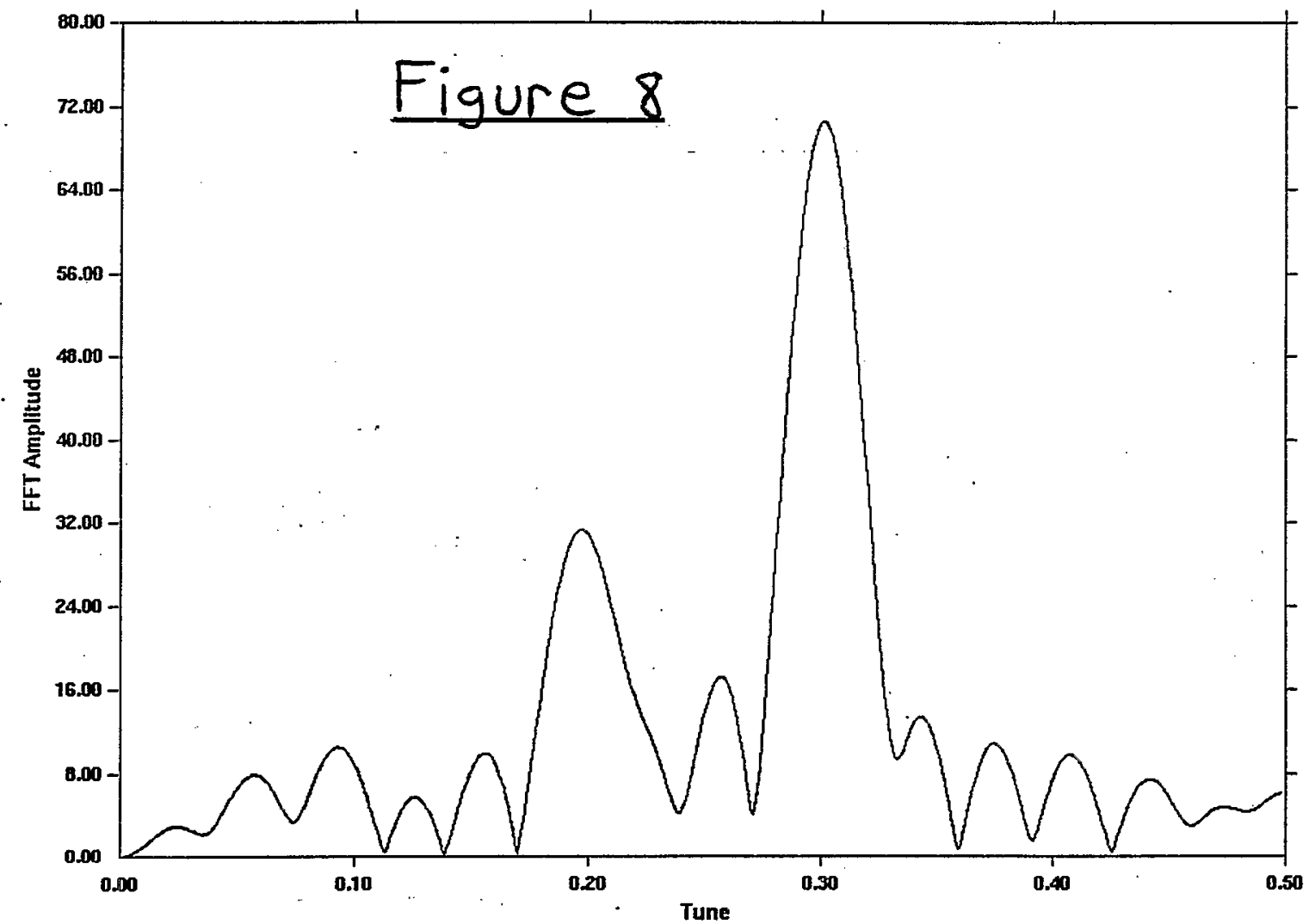


13-Apr-98

15:09:06

Figure 9

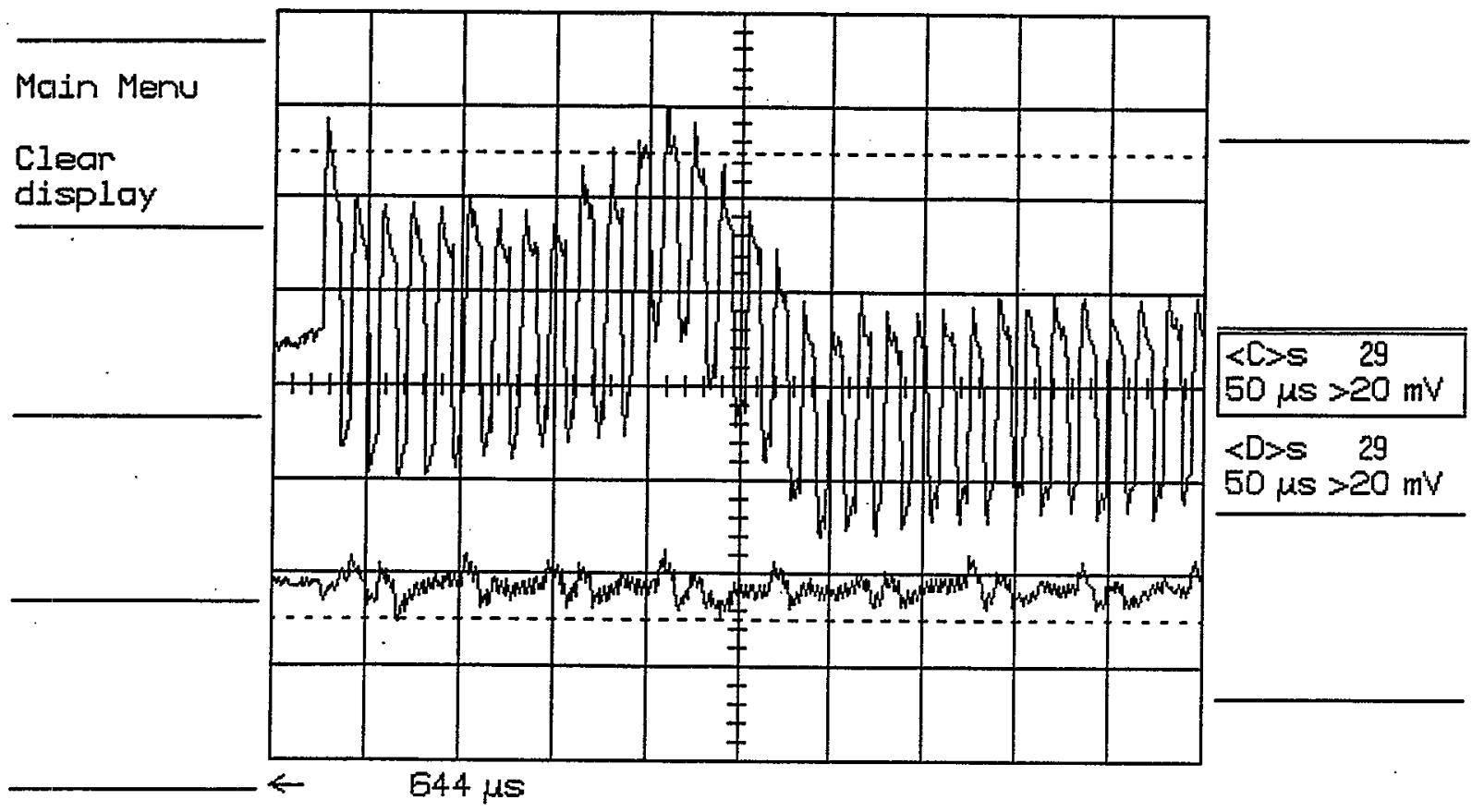

\section{Eigure 10}

SelectPue: Booster PUEHB4 $\_$Injection: Booster C3 Inflector $-\mid$

Mon Apr 13 15:05:39 1998

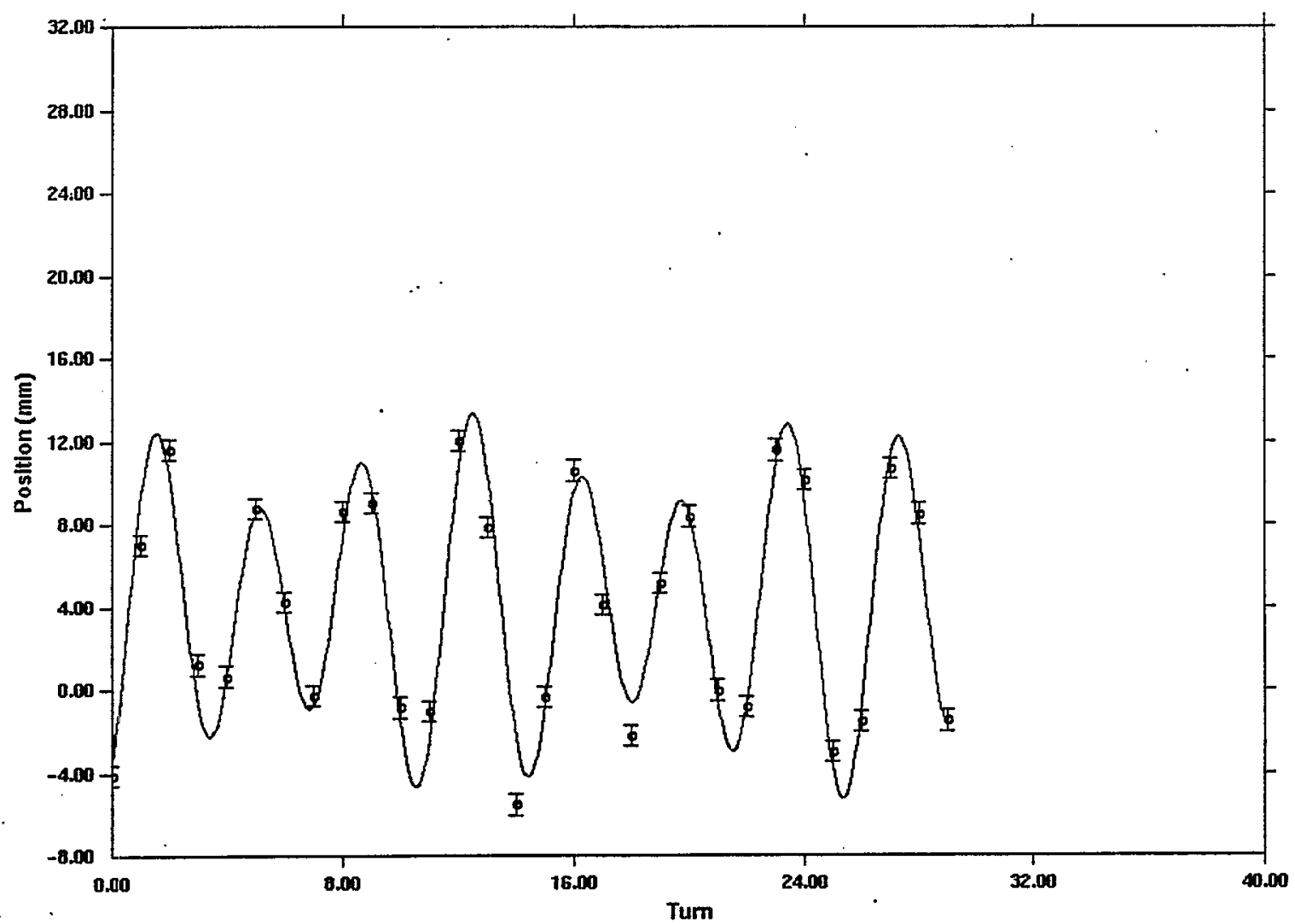


TRIGGER TIME :Date = APR 13, 1998, Time = 14:55:0.451

Fitted $\bar{P}$ arameters: Chisq/dof $=8.411162$, Number of Turns $=30$

$\mathrm{A} 1=0.272066+/-0.000365 \quad \mathrm{~A} 3=0.610808+/-0.006025$

$A 2=0.193830+/-0.001028 \quad A 4=0.562399+/-0.017388$

$A 6=2.377888+/-0.131103 \quad$ A. $=6.931011+/-0.128805$

$A 7=4.060254+/-0.181367$

$A 8=-0.000274+/-0.010710$

Period $=60.235294$ samples $=1.505882 \mathrm{e}-05$ seconds

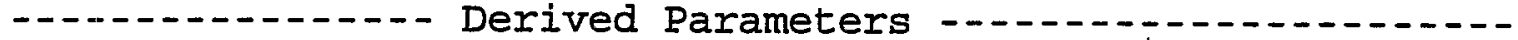

$\mathrm{Q} 1=4.7279+/-0.0004$

$\mathrm{Q} 2=4.8062+/-0.0010$

$\mathrm{AI}(\mathrm{mm})=6.93+/-0.13$

$\mathrm{A} 2(\mathrm{~mm})=2.38+/-0.13$

Epsilon 1 (mm mrad) $=5.989439+/-0.360084$

Epsilon 2 (mm mrad) $=1.153454+/-0.242061$

Position of eo at PUE (mm) $=4.06+/-0.18$

PUE : BOOSTER PUEHB4 INJ: BOOSTER C3_INFLECTOR

$\mathrm{H}$ Position wrt eo at PUE $(\mathrm{mm})=-7.52+7-0.25$

$\mathrm{H}$ Angle wrt eo at PUE (mrad) $=-1.530+/-0.050$

$\mathrm{V}$ Position wrt eo at PUE $(\mathrm{mm})=0.57+/-0.16$

$\mathrm{V}$ Angle wrt eo at PUE (mrad) $=0.242+/-0.083$

PUE: BOOSTER PUEHB4 INJ: BOOSTER C3 INFLECTOR

$\mathrm{H}$ Position wrt eo at INJ (mm) $=-7.94+7-0.21$

$H$ Angle wrt eo at INJ(mrad) $=-1.540+/-0.068$

- V Position wrt eo at INJ $(\mathrm{mm})=1.31+/-0.15$

$V$ Angle wrt eo at INJ (mrad) $=-0.109+/-0.087$

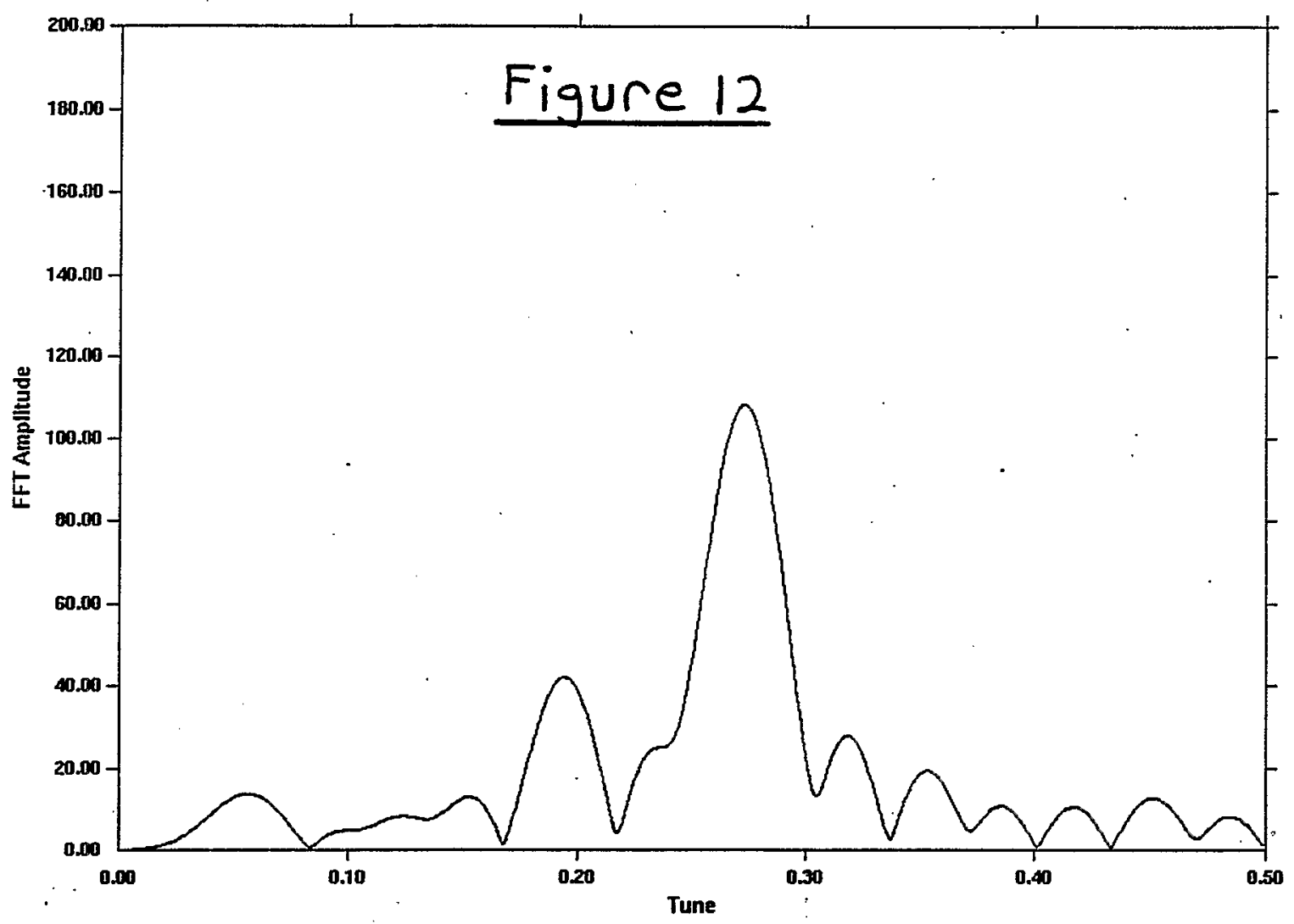


Figure 13. Scheme A, Layer 4

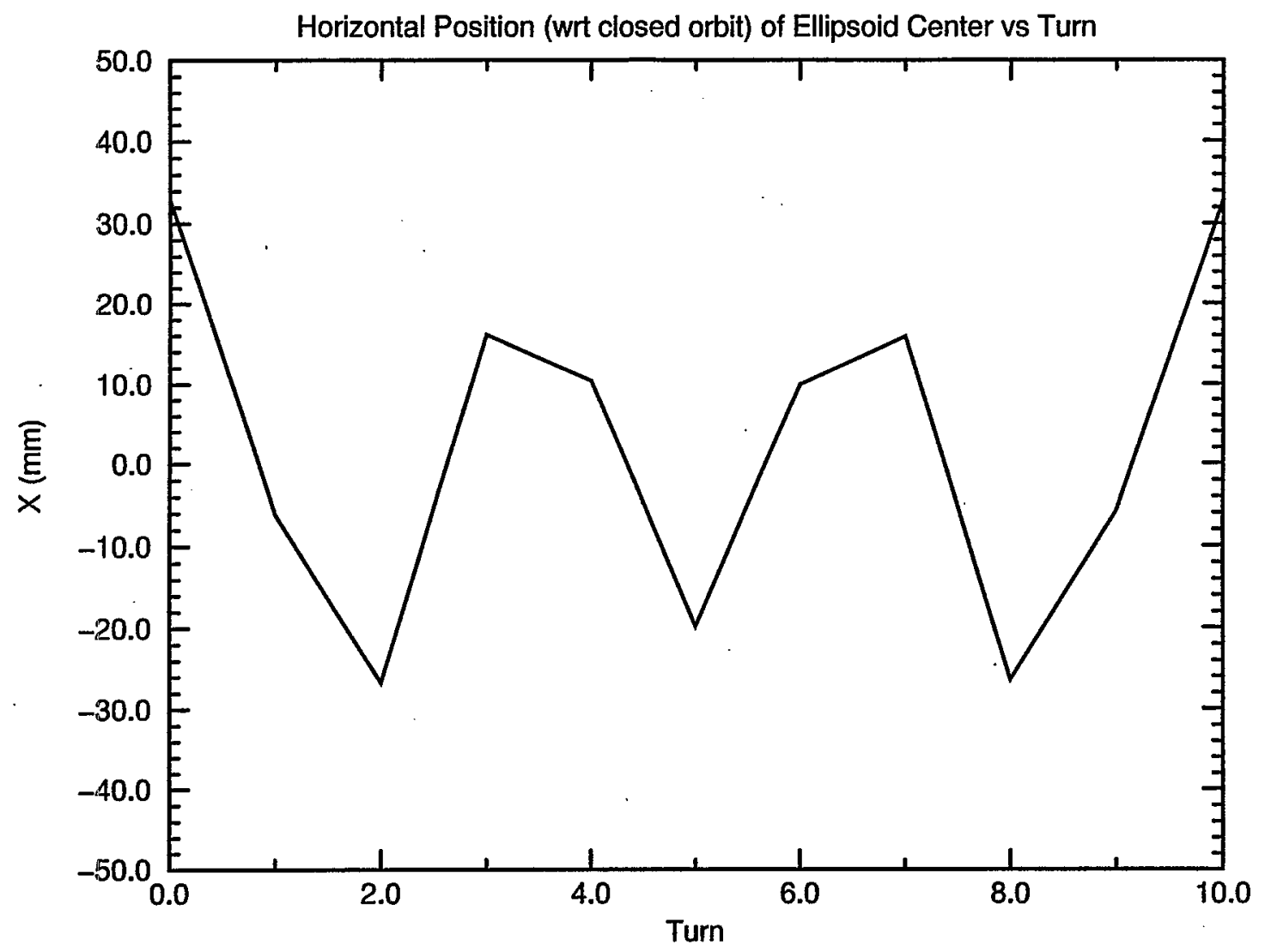

Figure 14. Scheme A, Layer 4

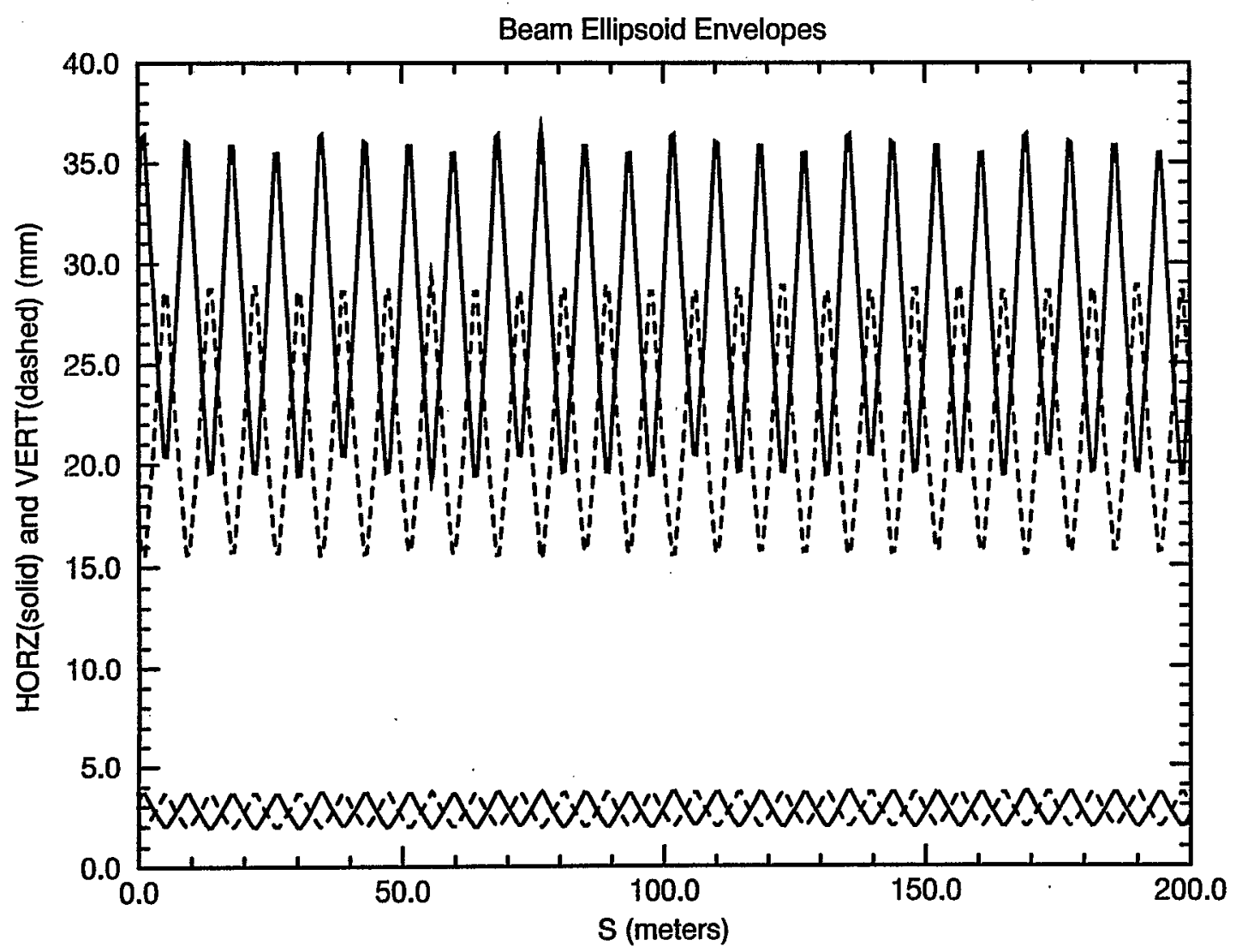


Figure 15. Scheme B, Layer 3

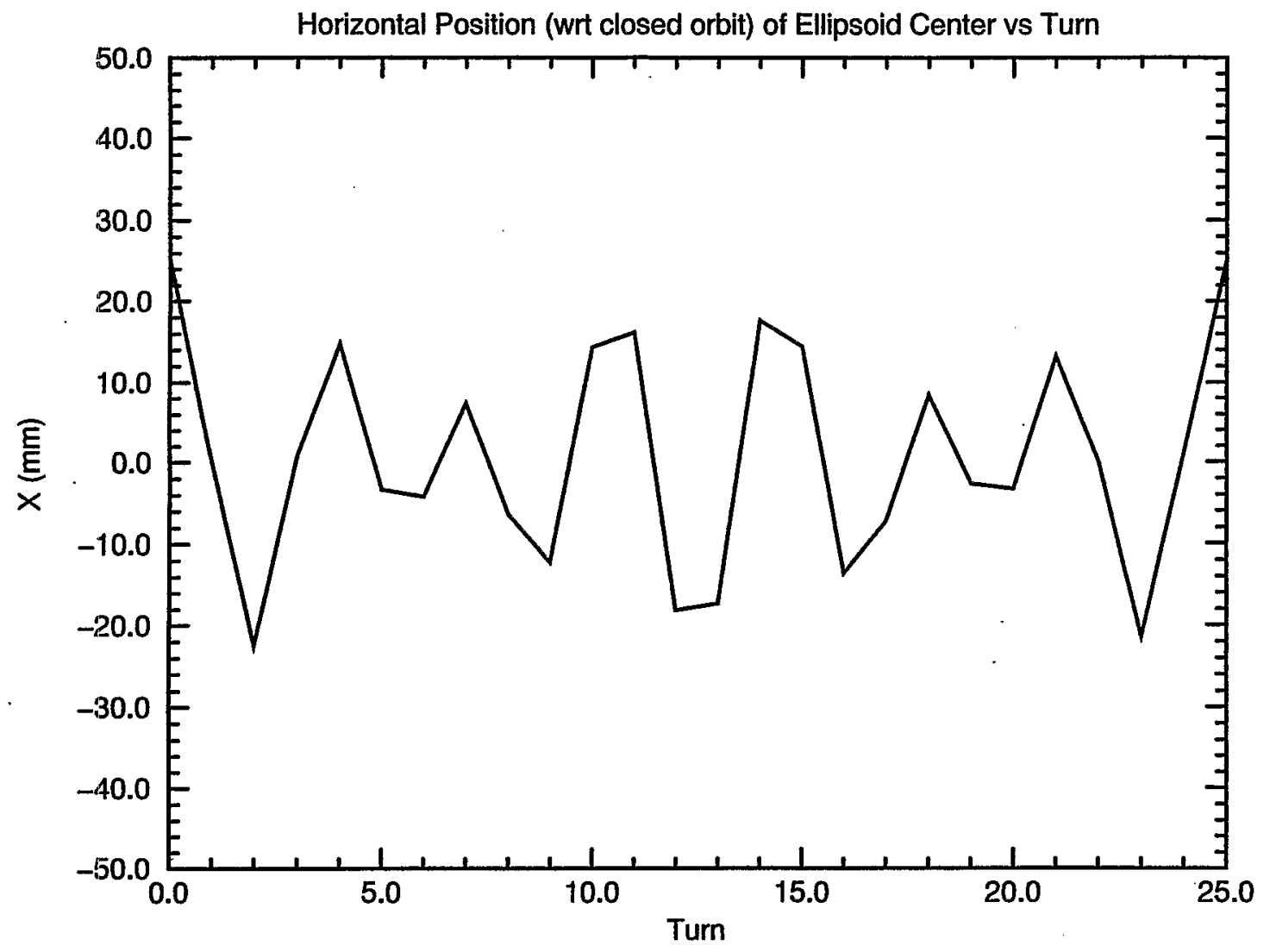

Figure 16. Scheme B, Layer 3

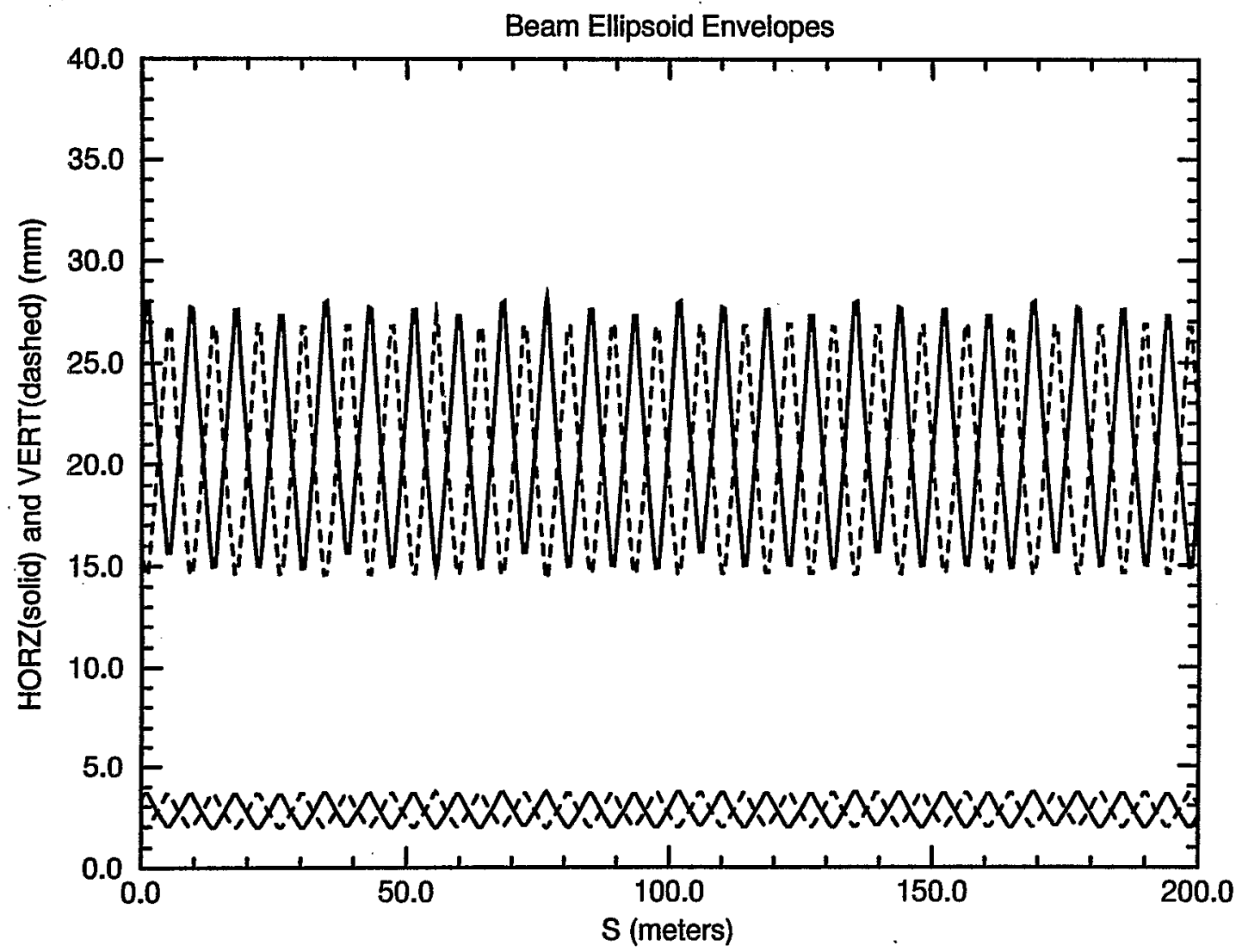

